\title{
K ATP Associated Developmental Delay, Epilepsy and Neonatal Diabetes
}

National Cancer Institute

\section{Source}

National Cancer Institute. KATP Associated Developmental Delay, Epilepsy and Neonatal

Diabetes. NCI Thesaurus. Code C131845.

A condition characterized by K ATP channel-associated permanent neonatal diabetes mellitus accompanied by neurological manifestations of developmental delay and epilepsy that may be associated with the severity of the mutation(s). 